\section{Radioembolization for colorectal liver metastases}

Maarten A. D. Vente, Maurice A. A. J. van den Bosch, Marnix G. E. H. Lam

and Johannes F. W. Nijsen

We read with great interest the recent Review by Nicolay et al. ${ }^{1}$ on yttrium-90 radioembolization $\left({ }^{90} \mathrm{Y}-\mathrm{RE}\right)$ in patients with unresectable colorectal metastases to the liver (Liver metastases from colorectal cancer: radioembolization with systemic therapy. Nat. Rev. Clin. Oncol. 6, 687-697; 2009). It provided the reader with a comprehensive overview of the current status of this relatively novel technique with respect to this type of cancer. However, we would like to take this opportunity to comment on a misinterpretation that the authors made when they discussed tumor response data from the literature. One of the articles the authors refer to in this section is a meta-analysis study on ${ }^{90} \mathrm{Y}-\mathrm{RE}$, authored by our group. ${ }^{2}$ In their discussion of this study, Nicolay et al..$^{1}$ state that we reported objective response rates (ORRs) of approximately $80 \%$ in patients treated in a salvage setting, and ORRs $>90 \%$ in patients who received radioembolization as a first-line treatment. This is incorrect since we did not assess ORR in our metaanalysis, which is defined as the portion of patients with complete or partial response, but instead the reported percentages refer to 'any response' (AR), which comprises the patients who exhibited complete response, partial response, and stable disease. We used AR instead of ORR because both partial response and stable disease were not uniformly defined in the studies that were included in the meta-analysis, mainly as a consequence of a difference between the Response Evaluation Criteria In Solid Tumors $\left(\right.$ RECIST) ${ }^{3}$ and WHO response criteria. ${ }^{4}$

Obviously, due to the combination of different response categories, AR derived from a series of studies will generally be higher than ORR. In fact, based on the raw data from our meta-analysis, ${ }^{2}$ we estimate ORRs for colorectal cancer metastatic to the liver to be approximately $80-90 \%$ in patients who received radioembolization as a first-line treatment, but only about $40 \%$ in patients treated in a salvage setting. That said, with respect to patients treated with ${ }^{90} \mathrm{Y}-\mathrm{RE}$ in both the first-line and in a salvage setting, response rate might actually be considerably higher because in most studies on ${ }^{90} \mathrm{Y}$-RE included in our meta-analysis, response was measured by cross-sectional anatomic imaging techniques (such as CT and MRI) that rely on tumor size measurement. However, it is known that lesion size does not always correlate with tumor response, due to several peritumoral and endotumoral processes that can occur post ${ }^{90} \mathrm{Y}-\mathrm{RE}$ treatment, such as peritumoral edema and necrosis. ${ }^{5}$

The latest RECIST criteria (RECIST 1.1 $)^{6}$ advise the use of PET using fluorine-18 fluorodeoxyglucose $\left({ }^{18} \mathrm{~F}-\mathrm{FDG}\right)$ for functional assessment of treatment response in ${ }^{18} \mathrm{~F}$-FDG-avid cancers, such as colorectal liver metastases. Most of the literature on ${ }^{90} \mathrm{Y}$-RE reports on tumor response following treatment, but one might argue that tumor response should not be the primary end point. Instead, we contend that progression-free interval and overall survival, as well as standardized quality-oflife measurements, are the preferred end points in comparative studies. Nicolay et al. ${ }^{1}$ mention the two large, ongoing, controlled trials, in which patients with colorectal liver metastases are randomly assigned to receive either systemic chemotherapy (consisting of oxaliplatin, 5-fluorouracil and leucovorin [FOLFOX]) or ${ }^{90} \mathrm{Y}$-RE with resin microspheres plus FOLFOX. The primary end points of these studies are progressionfree interval in the SIRFLOX $\operatorname{trial}^{7}$ and overall survival in the FOXFIRE trial. ${ }^{8}$ The results of these studies are expected to provide further insight into the true added value of this type of internal radiation therapy.

Department of Radiology and Nuclear Medicine, University Medical Center Utrecht, 100 Heidelberglaan, Utrecht 3584 CX, The Netherlands (M. A. D. Vente,

M. A. A. J. van den Bosch, M. G. E. H. Lam, J. F. W. Nijsen).

Correspondence to: M.A. D. Vente m.vente@umcutrecht.nl

\section{doi:10.1038/nrclinonc.2009.165-c1}

\section{Acknowledgments}

M. Wondergem, I. van der Tweel, B. A. Zonnenberg, and A. D. van het Schip are gratefully acknowledged for carefully proofreading this Correspondence.

Competing interests

The authors declare no competing interests.

1. Nicolay, N. H., Berry, D. P. \& Sharma, R. A. Liver metastases from colorectal cancer: radioembolization with systemic therapy. Nat. Rev. Clin. Oncol. 6, 687-697 (2009).

2. Vente, M. A. et al. Yttrium-90 microsphere radioembolization for the treatment of liver malignancies: a structured meta-analysis. Eur. Radiol. 19, 951-959 (2009).

3. Therasse, P. et al. New guidelines to evaluate the response to treatment in solid tumors. European Organization for Research and Treatment of Cancer, National Cancer Institute of the United States, National Cancer Institute of Canada. J. Natl Cancer Inst. 92, 205-216 (2000).

4. Park, J. O. et al. Measuring response in solid tumors: comparison of RECIST and WHO response criteria. Jpn J. Clin. Oncol. 33, 533-537 (2003).

5. Atassi, B. et al. Multimodality imaging following ${ }^{90} \mathrm{Y}$ radioembolization: a comprehensive review and pictorial essay. Radiographics 28, 81-99 (2008).

6. Eisenhauer, E. A. et al. New response evaluation criteria in solid tumors: revised RECIST guideline (version 1.1). Eur. J. Cancer 45, 228-247 (2009).

7. US National Library of Medicine. ClinicalTrials.gov [online], http://clinicaltrials.gov/ct2/show/ NCT00724503 (2010).

8. Sharma, R. A. et al. FOXFIRE: a phase III clinical trial of chemo-radio-embolisation as first-line treatment of liver metastases in patients with colorectal cancer. Clin. Oncol. (R. Coll. Radiol.) 20, 261-263 (2008). 